University of Nebraska - Lincoln

DigitalCommons@University of Nebraska - Lincoln

$5-1-2005$

\title{
Magnetic and electrical properties of single-phase multiferroic $\mathrm{BiFeO}_{3}$
}

Aswini K. Pradhan

Center for Materials Research, Norfolk State University, apradhan@nsu.edu

Kai Zhang

Center for Materials Research, Norfolk State University, Norfolk, Virginia

D. Hunter

Center for Materials Research, Norfolk State University, Norfolk, Virginia

J.B. Dadson

Center for Materials Research, Norfolk State University, Norfolk, Virginia

G.B. Loiutts

Center for Materials Research, Norfolk State University, Norfolk, Virginia

See next page for additional authors

Follow this and additional works at: https://digitalcommons.unl.edu/physicssellmyer

Part of the Physics Commons

Pradhan, Aswini K.; Zhang, Kai; Hunter, D.; Dadson, J.B.; Loiutts, G.B.; Bhattacharya, P.; Katiyar, R.; Zhang, Jun; Sellmyer, David J.; Roy, U.N.; Cui, Y.; and Burger, A., "Magnetic and electrical properties of singlephase multiferroic $\mathrm{BiFeO}_{3}$ " (2005). David Sellmyer Publications. 21.

https://digitalcommons.unl.edu/physicssellmyer/21

This Article is brought to you for free and open access by the Research Papers in Physics and Astronomy at DigitalCommons@University of Nebraska - Lincoln. It has been accepted for inclusion in David Sellmyer Publications by an authorized administrator of DigitalCommons@University of Nebraska - Lincoln. 


\section{Authors}

Aswini K. Pradhan, Kai Zhang, D. Hunter, J.B. Dadson, G.B. Loiutts, P. Bhattacharya, R. Katiyar, Jun Zhang, David J. Sellmyer, U.N. Roy, Y. Cui, and A. Burger 


\title{
Magnetic and electrical properties of single-phase multiferroic $\mathrm{BiFeO}_{3}$
}

\author{
A. K. Pradhan, ${ }^{\text {a) }}$ Kai Zhang, D. Hunter, J. B. Dadson, and G. B. Loutts \\ Center for Materials Research, Norfolk State University, 700 Park Avenue, Norfolk, Virginia 23504 \\ P. Bhattacharya and R. Katiyar \\ Department of Physics, University of Puerto Rico, San Juan, P.O. Box 23343, Puerto Rico 00931 \\ Jun Zhang and D. J. Sellmyer \\ Department of Physics and Astronomy and Center for Materials Research and Analysis, \\ University of Nebraska, Lincoln, Nebraska 68588-0113 \\ U. N. Roy, Y. Cui, and A. Burger \\ Department of Physics, Fisk University, 1000, 17 Avenue North, Nashville, Tennessee 37208
}

(Received 21 June 2004; accepted 3 February 2005; published online 18 April 2005)

\begin{abstract}
We have reported the structural, thermal, microscopic, magnetization, polarization, and dielectric properties of $\mathrm{BiFeO}_{3}$ ceramics synthesized by a rapid liquid-phase sintering technique. Optimum conditions for the synthesis of single-phase $\mathrm{BiFeO}_{3}$ ceramics were obtained. Temperature-dependent magnetization and hysteresis loops indicate antiferromagnetic behavior in $\mathrm{BiFeO}_{3}$ at room temperature. Although saturated ferroelectric hysteresis loops were observed in single-phase $\mathrm{BiFeO}_{3}$ ceramic synthesized at $880{ }^{\circ} \mathrm{C}$, the reduced polarization is found to be due to the high loss and low dielectric permittivity of the ceramic, which is caused by higher leakage current. () 2005 American Institute of Physics. [DOI: 10.1063/1.1881775]
\end{abstract}

\section{INTRODUCTION}

Multiferroics are a class of materials with coupled electric, magnetic, and structural order parameters that yield simultaneous effects of ferroelectricity, ferromagnetism, and ferroelasticity in the same material. ${ }^{1}$ These materials offer a wide opportunity for potential applications in information storage, such as spintronic devices and sensors, where both electric and magnetic polarizations can be coupled, giving enough opportunity for manipulating devices. The perovskite $\mathrm{BiFeO}_{3}$ (BFO) belongs to this class of multiferroic materials with ferroelectric transition temperature $T_{c} \sim 1103 \mathrm{~K}$ and antiferromagnetic transition $T_{N} \sim 643 \mathrm{~K}$. BFO belongs to other magnetic ferroelectrics, such as $\mathrm{YMnO}_{3}$ and $\mathrm{BiMnO}_{3}$. Bulk $\mathrm{BFO}$, especially synthesized through solid solution, exhibits weak ferromagnetic properties at room temperature due to residual moment from a canted spin structure, ${ }^{2}$ which is believed to be generated from the impurity phase related to $\mathrm{Fe}$ phase separation. In addition, a major problem generally encountered in transport measurements is the high leakage current due to the low resistivity of the sample, which creates difficulties in the observation of the ferroelectric hysteresis loops. The high leakage current in bulk is attributed to defects and nonstoichiometry. Therefore, this material becomes unsuitable for applications. However, there is tremendous success in the fabrication of heteroepitaxially constrained thin films of BFO which shows enhanced polarization with saturated hysteresis loop and ferromagnetic behavior at room temperature. ${ }^{3,4}$

There are several reports ${ }^{5,6}$ on the fabrication of BFO dispersed in $\mathrm{BaTiO}_{3}$ matrix to restrict the second phase, leak-

\footnotetext{
${ }^{\text {a) }}$ Author to whom correspondence should be addressed: electronic mail: apradhan@nsu.edu
}

age current, and low resistivity in the sample. Recently, single-phase BFO ceramic samples have been synthesized by rapid liquid-phase sintering technique. ${ }^{7}$ These ceramic samples show relatively high resistivity and saturated ferroelectric hysteresis loops at room temperature and could be useful for potential applications. This is a dramatic improvement over past techniques used for the fabrication of BFO ceramics in which very often canted ferromagnetic spin structures were observed due to the impurity phase. However, detailed studies on microscopic grains, thermal, magnetic, and electrical properties related to frequencydependent dielectric loss are still lacking in this potentially important ceramic sample. In this paper, we report on the development of various microstructures of liquid-phasesintered samples processed at various temperatures, thermal properties, temperature-dependent magnetic behavior, and electrical properties including ferroelectric loops and related dielectric loss up to $f=1 \mathrm{MHz}$. Our results suggest that pure BFO samples exhibit antiferromagnetic behavior over the whole temperature region, 5-300 K, and saturated ferroelectric loops at room temperature. However, the ceramic sample shows high dielectric loss, especially at higher frequency range, which may be one of the major challenges for applications.

\section{EXPERIMENT}

$\mathrm{BiFeO}_{3}$ samples were synthesized by the solid-state reaction method. Stoichiometric amounts of $\mathrm{Bi}_{2} \mathrm{O}_{3}(99.99 \%)$ and $\mathrm{Fe}_{2} \mathrm{O}_{3}$ (99.99\%) powders were thoroughly mixed using isopropyl alcohol and ground. The mixtures were dried, pressed, and sintered at different temperatures for 400-450 s, as described in Ref. 7 for rapid liquid-phase sintering. Phase identification was performed by the x-ray powder diffraction 


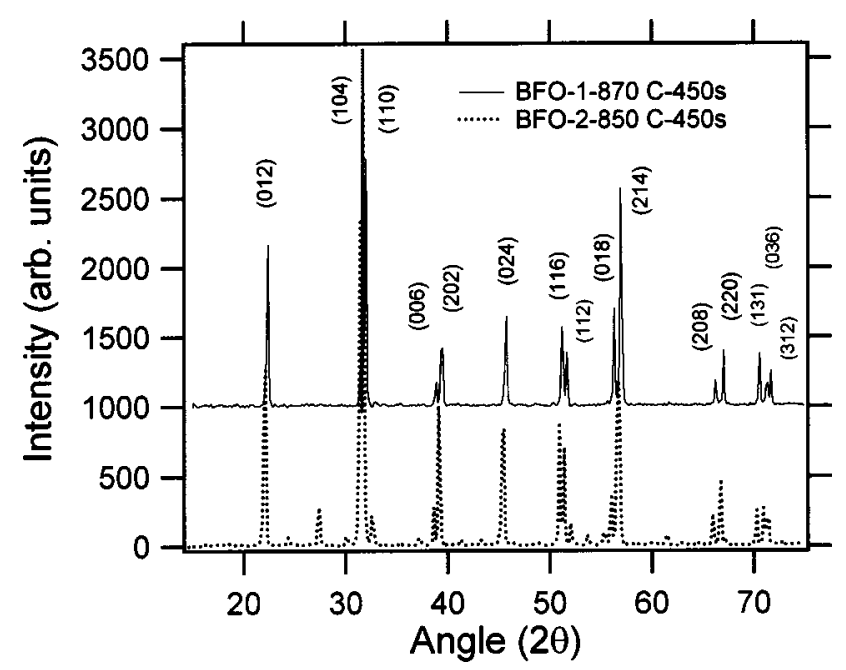

FIG. 1. X-ray diffraction pattern of $\mathrm{BiFeO}_{3}$ ceramics synthesized at 820 and $880{ }^{\circ} \mathrm{C}$ by liquid-phase sintering. The asterisks represent the undesired second phase.

(XRD) method on a Rigaku x-ray diffractometer using $\mathrm{Cu} K \alpha$ radiation. The magnetization was measured using Quantum Design superconducting quantum interference device [SQUID magnetic property measurement system (MPMS)]. RT6000 ferroelectric tester and Agilent dielectric bridge measured the ferroelectric and dielectric properties of the sintered ceramics at room temperature, respectively.

\section{RESULTS AND DISCUSSION}

Figure 1 shows the XRD patterns of the BFO samples sintered at $850-880{ }^{\circ} \mathrm{C}$, respectively, prepared by liquidphase sintering for $\sim 450 \mathrm{~s}$. The sample sintered at $880^{\circ} \mathrm{C}$ shows a single phase, whereas the sample sintered at $850{ }^{\circ} \mathrm{C}$ shows evidence for the presence of a second phase, probably arising from $\mathrm{Bi}_{36} \mathrm{Fe}_{2} \mathrm{O}_{57}$ and related phases. Hence the rapid sintering at $880{ }^{\circ} \mathrm{C}$ for a short time shows a completion of reaction to form a single-phase $\mathrm{BiFeO}_{3}$. It is noted that the sintering temperature higher than that of $880{ }^{\circ} \mathrm{C}$, such as at $890{ }^{\circ} \mathrm{C}$, forms many undesirable phases. In order to investigate the thermal behavior related to phase changes and melting phenomena in BFO, differential scanning calorimetry (DSC) results are presented in Fig. 2. A distinct endothermic transition at $825^{\circ} \mathrm{C}$ in heat flow versus temperature curve is observed. This transition is very consistent with the change in curvature in heat-flow curve, as shown in Fig. 2 as dashed lines. The melting point of $\mathrm{Bi}_{2} \mathrm{O}_{3}$ is about $825^{\circ} \mathrm{C}$ and this transition can be certainly assigned due to the melting of $\mathrm{Bi}_{2} \mathrm{O}_{3}$. Therefore, it remains very important at what temperature the liquid phase of $\mathrm{Bi}_{2} \mathrm{O}_{3}$ forms a single-phase material with $\mathrm{Fe}_{2} \mathrm{O}_{3}$. It turns out that $880{ }^{\circ} \mathrm{C}$ becomes the exact temperature for rapid sintering where single-phase $\mathrm{BFO}$ is formed. On the other hand, there is a remarkable weight loss after the second transition at $960{ }^{\circ} \mathrm{C}$, indicating loss of $\mathrm{Bi}_{2} \mathrm{O}_{3}$ through sublimation process. Hence rapid heating rate and short-time sintering of $\mathrm{BiFeO}_{3}$ at $880{ }^{\circ} \mathrm{C}$ remains a clue to avoid loss of $\mathrm{Bi}$, segregation of $\mathrm{Fe}_{2} \mathrm{O}_{3}$, and formation of other undesired phases, as indicated by the XRD. Lower-

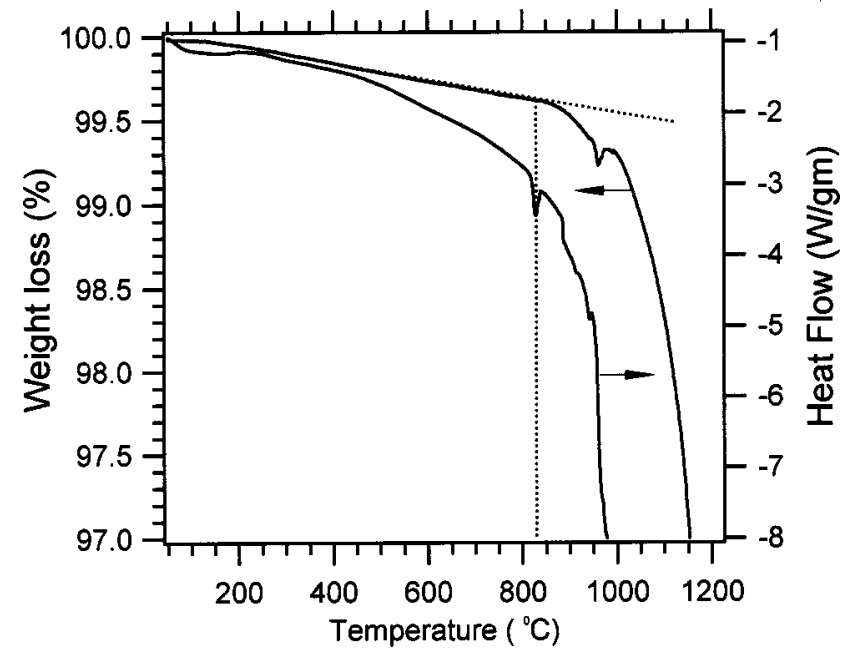

FIG. 2. Temperature dependence of weight loss and heat flow of BFO measured by differential scanning calorimetry.

temperature rapid sintering is also unfavorable due to the incomplete melting of $\mathrm{Bi}_{2} \mathrm{O}_{3}$ and the formation of intermediate phases.

Figure 3 shows the scanning electron microscopy (SEM) results of BFO synthesized by rapid thermal sintering at 850 , 880 , and $890^{\circ} \mathrm{C}$. The SEM micrographs elucidate the grain growth phenomena in sintered BFO at various temperatures. The grain size increases enormously with increasing temperature. The average grain of $1-2 \mu \mathrm{m}$ increases to $5-10 \mu \mathrm{m}$ in size when sintering temperature increases from 850 to $880{ }^{\circ} \mathrm{C}$. However, the density and number of bright spots that probably correspond to segregated $\mathrm{Fe}_{2} \mathrm{O}_{3}$ particles decreases dramatically when sintering temperature increases from 850 to $880{ }^{\circ} \mathrm{C}$. In fact, several red-colored spots embedded in a black matrix were noticed when examined in an optical microscope. This suggests that the bright spots observed in SEM are due to $\mathrm{Fe}_{2} \mathrm{O}_{3}$ particles. On the other hand, the SEM micrograph of the sample sintered at $890{ }^{\circ} \mathrm{C}$ shows a uniform surface displaying very large grains $(>50 \mu \mathrm{m})$. However, this sample is multiphase due to partial loss of $\mathrm{Bi}_{2} \mathrm{O}_{3}$ from its melt, as discussed in X-ray and DSC studies. This suggests that although large grains are produced by melting, the grains contain many undesired phases.

In order to illustrate the magnetic ordering in BFO, the temperature dependence of zero-field-cooled (ZFC) and field-cooled (FC) magnetization curves are presented in Fig. 4(a). In these measurements, the sample was cooled in either ZFC to a desired temperature and then a magnetic field was applied or FC. The measurements were done while warming the sample in the magnetic field for both cases. For $H$ $=0.2 \mathrm{~T}$, the $\mathrm{FC}$ and $\mathrm{ZFC}$ curves separate at around $160 \mathrm{~K}$ and $M_{\mathrm{FC}}-M_{\mathrm{ZFC}}$ increases as temperature decreases. However, the FC and ZFC curves again merged at very low temperatures. There is a distinct irreversibility at $\sim 160 \mathrm{~K}$ accompanied by a remarkable thermomagnetic hysteresis (or irreversibility) with $M_{\mathrm{ZFC}} \neq M_{\mathrm{FC}}$ at the irreversibility temperature $T_{\text {irr }}$. This type of irreversibility in magnetization is generally indicative of antiferromagnetic (AF) ordering; ${ }^{8}$ however, for a simple AF no thermomagnetic hysteresis is expected. This latter effect is traditionally accepted as a hall- 

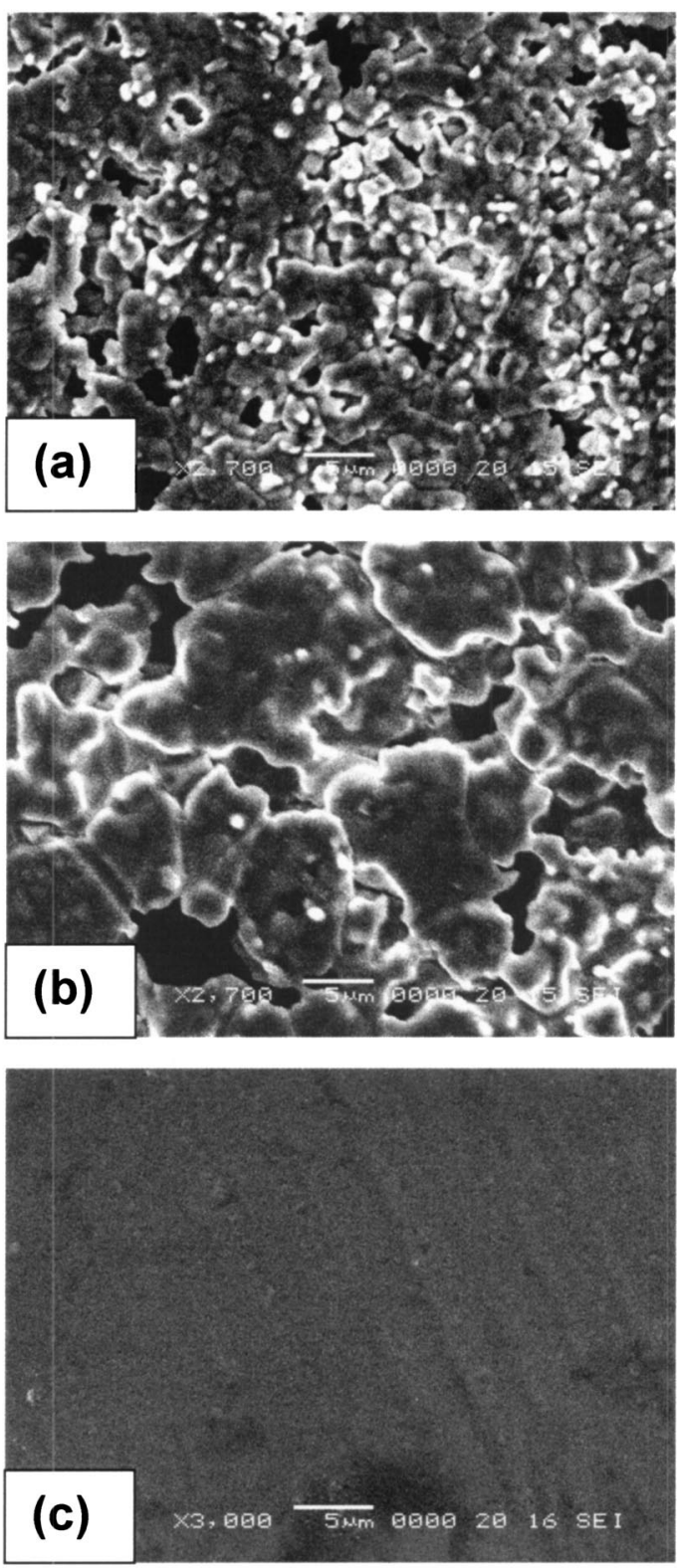

FIG. 3. Scanning electron microscopic images of BFO ceramics synthesized at (a) 820 , (b) 880 , and (c) $890^{\circ} \mathrm{C}$ by rapid liquid-phase sintering. The indicated scale in all images is $5 \mu \mathrm{m}$.

mark of a spin-glass system ${ }^{9}$ with a characteristic spin-glass transition temperature $T_{g}$. The most interesting phenomenon is the observation of an anomalous temperature dependence of magnetization, such as a decrease in magnetization down to $150 \mathrm{~K}$ accompanied by a small jump at about $265 \mathrm{~K}$, and then a sharp increase of magnetization, as the temperature is further lowered. Although the latter temperature-dependent magnetization behavior is understood in the framework of antiferromagnetic correlation, the decrease of magnetization with temperature in the temperature range of from 265 to about $150 \mathrm{~K}$ remains puzzling and could not be explained.

One of the suspects for such anomalous magnetization may be the oxygen vacancy concentration and also the existence of $\mathrm{Fe}^{2+}$ ions. It was proposed ${ }^{4}$ that the oxygen deficiency may lead to valence fluctuation of $\mathrm{Fe}$ ions $3+$ to $2+$ states) in $\mathrm{BFO}$ resulting in high conductivity. However, such
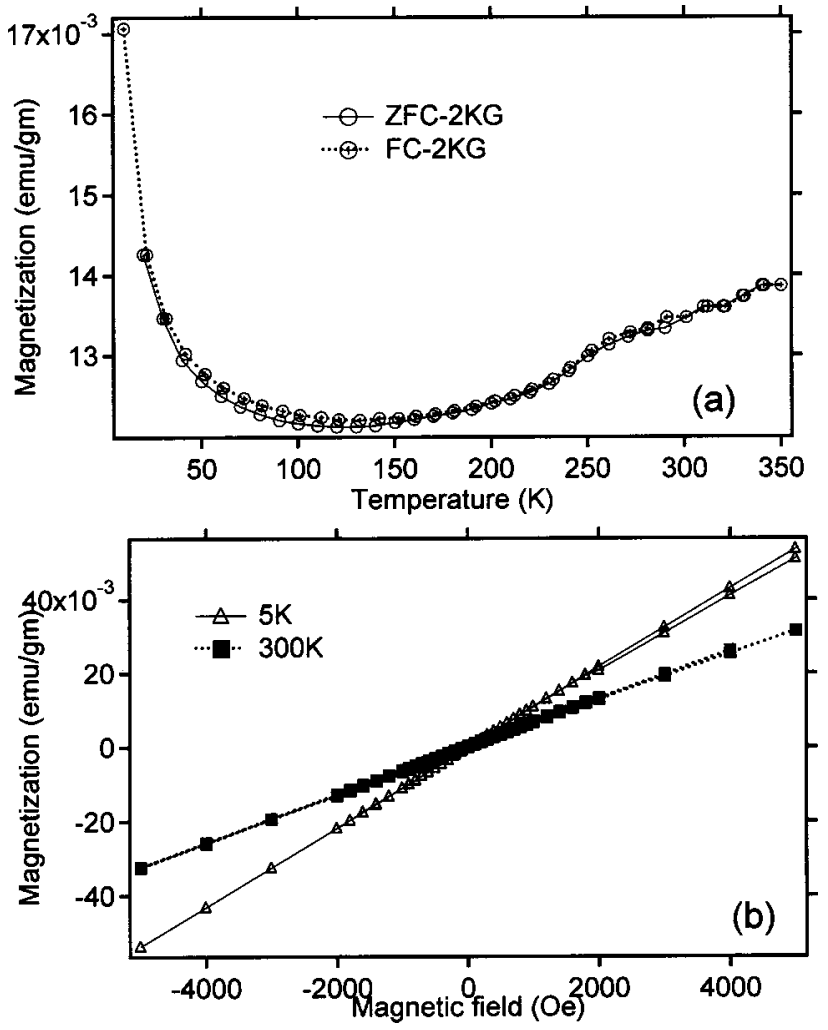

FIG. 4. (a) The temperature-dependent FC and ZFC magnetizations of BFO ceramic synthesized by liquid-phase sintering at $880^{\circ} \mathrm{C}$. (b) The magnetization hysteresis loops of BFO at 5 and $300 \mathrm{~K}$.

a possibility was recently ruled out in samples synthesized by liquid-phase sintering ${ }^{7}$ where sintering time for the heating, sintering, and cooling times were limited to seconds only. We argue that even though the sintering time is considerably small, there may exist small concentrations of oxygen vacancies resulting in $\mathrm{Fe}^{2+}$ and $\mathrm{Fe}^{3+}$ valence fluctuations which may influence significantly both magnetic and electrical properties. Hence, the observed anomalous magnetization behavior may be related to such valence fluctuations, although further experiments are necessary to reveal the complete fact. However, the magnetic hysteresis loops, as shown in Fig. 4(b), illustrate a linear field dependence, indicating that $\mathrm{BFO}$ shows antiferromagnetic behavior both at 5 and $300 \mathrm{~K}$. On the other hand, the magnetization and magnetic hysteresis results confirm the absence of canted ferromagnetic behavior in this sample. This suggests the absence of Fe-related clusters or impurities in the sample.

The ferroelectric polarization hysteresis loops are presented in Fig. 5 for the BFO sample sintered at $880{ }^{\circ} \mathrm{C}$. According to previous reports, ${ }^{2,10}$ the spontaneous polarization in BFO ceramics and single crystals is significantly lower than the expected value for other ferroelectric materials having comparable $T_{c}$, such as $\mathrm{PbTiO}_{3}$ with $T_{c} \sim 763 \mathrm{~K}$, and spontaneous polarization of $80-100 \mu \mathrm{C} / \mathrm{cm}^{2}$. The spontaneous polarization $\left(P_{s}\right)$, remnant polarization $\left(R_{s}\right)$, and the coercive field $\left(E_{c}\right)$ are about $3.5 \mu \mathrm{C} / \mathrm{cm}^{2}, 2.5 \mu \mathrm{C} / \mathrm{cm}^{2}$, and 40 $\mathrm{kV} / \mathrm{cm}$, respectively. Although these values are very comparable to the values reported in the single crystal, ${ }^{10}$ they are almost half of the reported values for BFO ceramics synthesized using the same technique. However, a saturated polar- 


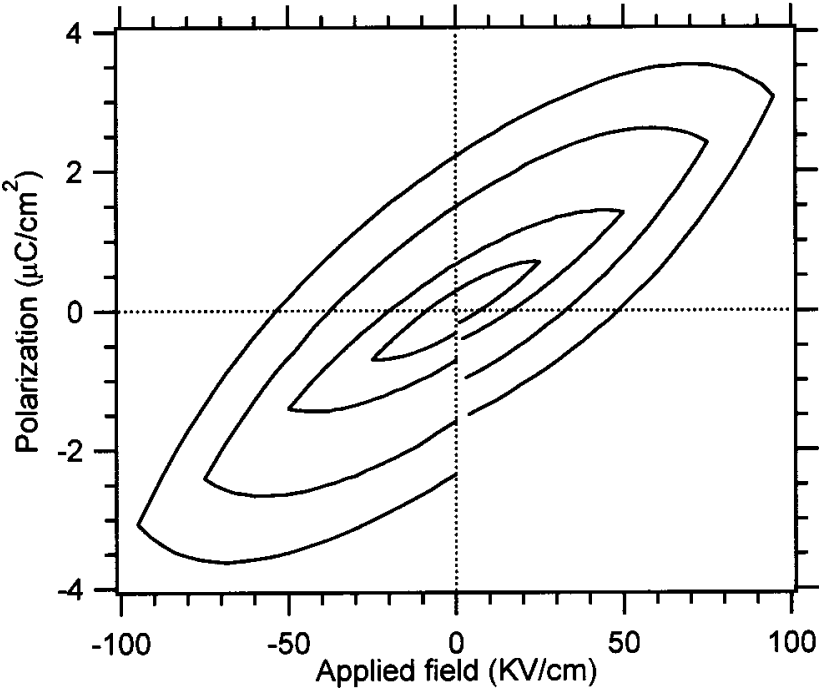

FIG. 5. Polarization hysteresis loops of BFO ceramic synthesized by liquidphase sintering at $880^{\circ} \mathrm{C}$ for different field cyclings.

ization hysteresis loop was observed at room temperature under an applied field of $95 \mathrm{kV} / \mathrm{cm}$. In order to understand the origin of such low values for $P_{s}, R_{s}$, and $E_{c}$, the dielectric constant and the loss as a function of frequency are presented in Fig. 6. It is clear from Fig. 6 that the dielectric constant is a strong function of frequency and it decreases significantly with increasing frequency. However, a reverse trend is obtained for the dielectric loss factor, $\tan \delta$. The high loss in this material generally originated from the higher conductiv-

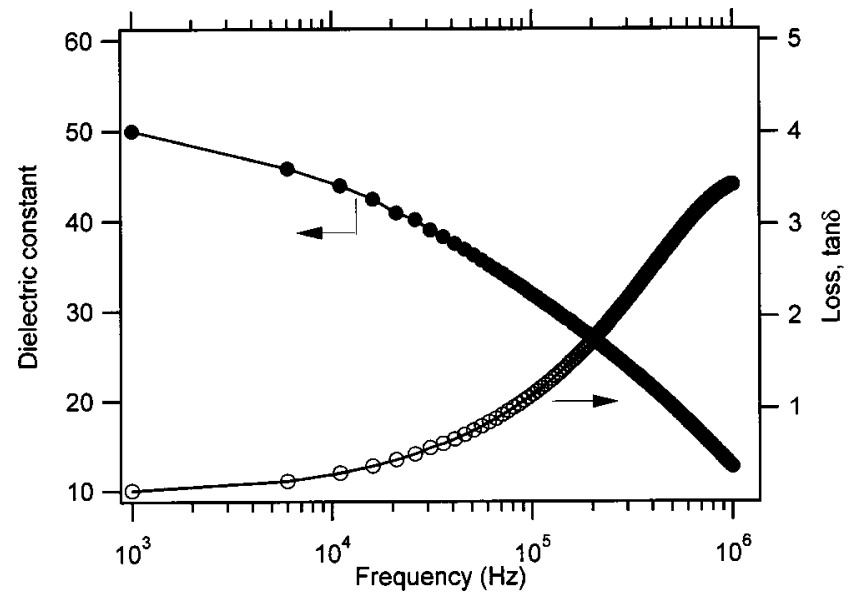

FIG. 6. Frequency dependence of the dielectric constant and loss of the BFO ceramic synthesized by liquid-phase sintering at $880{ }^{\circ} \mathrm{C}$. ity causing higher leakage current. This is one of the major reasons for obtaining lower values for $P_{s}, R_{s}$, and $E_{c}$ in BFO pure materials. Therefore, it is necessary to synthesize BFO in other dielectric matrix to enhance its dielectric and loss properties in order to realize its practical applications.

\section{CONCLUSION}

We have reported the $\mathrm{x}$-ray, thermal, microscopic, magnetization, polarization, and dielectric properties of $\mathrm{BiFeO}_{3}$ ceramics synthesized by a rapid liquid-phase sintering technique. Temperature-dependent magnetization and hysteresis loops indicate antiferromagnetic behavior in $\mathrm{BiFeO}_{3}$ at room temperature. Anomalous temperature-dependent magnetization behavior predicts the presence of a small concentration of oxygen-related defects due to Fe valence fluctuations. Although saturated ferroelectric hysteresis loops were observed in single-phase $\mathrm{BiFeO}_{3}$ ceramic synthesized at $880{ }^{\circ} \mathrm{C}$, the reduced polarization is found to be due to the high loss and low dielectric permittivity of the ceramic. In the present paper, we have exclusively demonstrated the multiphasesingle-phase-multiphase transition in $\mathrm{BiFeO}_{3}$ ceramics with increasing sintering temperature and their structural, thermal, and microscopic evolutions. The persisting leakage current problem in optimally sintered ceramics needs to be solved for better applications.

\section{ACKNOWLEDGMENTS}

This work is supported by the National Aeronautics and Space Administration (NASA) and National Science Foundation (NSF) for Center for Research Excellence in Science and Technology (CREST) Grant No. HRD-9805059. Research at the University of Nebraska is supported by NSFMRSEC, ONR, and CMRA.

${ }^{1}$ E. K. H. Salje, Phase Transitions in Ferroelectrics, Ferroelastic and Coelastic Crystals (Cambridge University Press, Cambridge, 1990).

${ }^{2}$ G. A. Smolenski, Sov. Phys. Usp. 25, 475 (1982).

${ }^{3}$ J. Wang et al., Science 299, 1719 (2003).

${ }^{4}$ V. R. Palkar, J. John, and R. Pinto, Appl. Phys. Lett. 80, 1628 (2002).

${ }^{5}$ K. Ueda, H. Tabata, and T. Kawai, Appl. Phys. Lett. 75, 555 (1999).

${ }^{6}$ M. Mahesh Kumar, A. Srinivas, and S. V. Suryanarayan, J. Appl. Phys. 87, 855 (2000).

${ }^{7}$ Y. P. Wang, L. Zhou, M. F. Zhang, X. Y. Chen, J.-M. Liu, and Z. G. Liu, Appl. Phys. Lett. 84, 1731 (2004).

${ }^{8}$ N. Hill and N. A. Spaldin, Magnetic Materials (Cambridge University Press, Cambridge, 2003).

${ }^{9}$ K. H. Fischer, J. A. Hertz, and D. Edwards, Spin Glasses (Cambridge University Press, Cambridge, 1991).

${ }^{10}$ J. R. Teague, R. Carson, and W. J. James, Solid State Commun. 8, 1073 (1970). 